\title{
A Survey of Machine Translation Techniques and Systems for Indian Languages
}

\author{
Sandeep Saini \\ Department of Electronics and Communication Engineering \\ Malviya National Institute of Technology \\ Jaipur, India \\ sandeep.saini@1nmiit.ac.in
}

\author{
Vineet Sahula \\ Department of Electronics and Communication Engineering \\ Malviya National Institute of Technology \\ Jaipur, India \\ sahula@ieee.org
}

\begin{abstract}
Machine Translation pertains to translation of one natural language to other by using automated computing. The main objective is to fill the language gap between two different languages speaking people, communities or countries. In India, we have multiple and hugely diverse languages and scripts, hence scope and need of language translation is immense. In this paper, we focus on the current scenario of research in machine translation in India. We have reviewed various important Machine Translation Systems (MTS) and presented preliminary comparison of the core methodology as used by them.
\end{abstract}

Keywords-Natural language processing, Learning systems, Statistical Machine Translation.

\section{INTRODUCTION}

Machine Translation (MT) is a sub-field of computational linguistics that investigates the use of computer software to translate text or speech from one natural language to another [1] . At its basic level, MT performs simple substitution of words in one natural language for words in another. Machine Translation system are needed to translate literary works which from any language into native languages. The literary work is fed to the MT system and translation is done. Such MT systems can break the language barriers by making available work rich sources of literature available to people across the world. MT also overcomes the technological barriers. Most of the information available is in English which is understood by only 3\% of the population [2]. This has led to digital divide in which only small section of society can understand the content presented in digital format. MT can help in this regard to overcome the digital divide.

In a large multilingual society like India, there is great deman for translation of documents from one language to another language. There are 22 constitutionally approved languages, which are officially used in different states. There are about 1650 dialects spoken by different communities. There are 10 Indic scripts. All of these languages are well developed and rich in content. They have similar scripts and grammars. The alphabetic order is also similar. Some languages use common script, especially Devanagari. Hindi written in the Devanagri script is the official language of the union Government. English is also used for government notifications and communications. Indian average literacy level is 65.4 percent (Census 2001). Less than 5 per cent of people can either read or write English.
Moreover, over 95 percent of the population is normally deprived of the benefits of Information Technology due to language barrier [3]. All these make language translation a necessary one. Work in the area of Machine Translation in India has been going on for several decades. During the early 90s, advanced research in the field of Artificial Intelligence and Computational Linguistics made a promising development of translation technology. This helped in the development of usable Machine Translation Systems in certain well-defined domains.

Fully automatic high quality machine translation system (FGH_MT) is extremely difficult to build. In fact there is no system in the world which qualifies to be called FGH_MT. Many organizations like IIT(Kanpur), CDAC(Mumbai), CDAC(Pune), IIIT(Hyderabad) etc. are engaged in development of MT systems under projects sponsored by Department of Electronics (DoE), state governments etc. since 1990 [4]. Translation between structurally similar languages like Hindi and Punjabi is easier than that between language pairs that have wide structural difference like Hindi and English., Translation systems between closely related languages are easier to develop since they have many parts of their grammars and vocabularies in common [5]. Research on MT systems between Indian and foreign languages and also between Indian languages are going on in these institutions.

Research on Indian language translation is extensively carried forward in many research centres outside India as well. Anoop Sarkar and group at Simon Fraser University is actively working on Hierarchical Phrase-based Translation. Kriya [6] is an implementation of hierarchical phrase-based (hiero) SMT system. Dr Raman et. al at GWU, Washington DC have carried forwarded collaborative research with IIT Madras to develop an AI-based approach to machine translation in Indian languages [7]. Most of the MT systems are evaluated for their accuracy by its BLEU score. This method for automatic evaluation of machine translation was proposed by Papineni [8] and accepted worldwide now.

In this paper we focus on discussing different approaches for Machine Translations adopted for Indian languages and their features. In section II we discuss about different approaches for MT systems. In section III different machine translation systems for Indian languages are compared. We conclude our study in section IV. 


\section{APPROACHES FOR MACHINE TRANSLATION}

Mandelblit, in his article "Machine translation: a cognitive linguistics approach" [9], describes four different but complementary "memories" have been identified by the researchers for their work:

1) knowledge of the language system;

2) knowledge of language usage;

3) knowledge of the world; and

4) knowledge of the situation.

Human translators use all four types of memory. Machine Translation systems use some (but not all) of them. Current MT systems all rely on the first type, i.e. grammatical and lexical competence, and vary with regard to their integration of the third type, i.e. world-knowledge bases. Contextual knowledge (knowledge of the situation) has come to be recognized as an important factor and is currently under investigation.

Machine Translation (MT) systems have limitations and reservations in using all four type of memories. Cognitive approach is the main hindrance in developing an accurate MT system. These systems can be classified according to the basic approach by which they perform translation. A classification of MT approaches is given in Figure 1. The MT systems can also be classified according to use of traditional or modern technology.

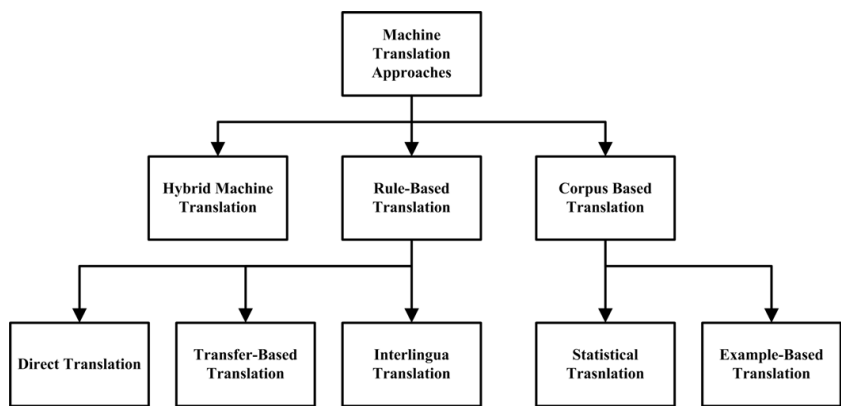

Fig. 1. Different Machine Translation Approaches

\section{A. Rule Based Machine Translation}

Rule Based Machine Translation (RBMT), also known as Knowledge Based Machine Translation is classical approach to machine translation. In rule-based systems, the source text is parsed and an intermediate representation is produced. The target language text is generated from the intermediate representation. The systems are based on linguistic information about source and target languages. This information is basically derived from grammars and dictionaries covering the main semantic, morphological, and syntactic regularities of each language respectively. RBMT is further classified as Direct Translation, Interlingua based Machine Translation and Transfer based Machine Translation depending on the intermediate representation.

1) Direct Machine Translation: Direct Machine Translation is the one of the simplest machine translation approach. In Direct Machine Translation, a direct word by word translation of the input source is carried out with the help of a bilingual dictionary and after which some syntactical rearrangement are made. Figure 2 explains the concept of Direct translation.

In Direct Machine Translation a language called the source language is given as input and the output is known as the target language. Typically, the approach is unidirectional and only takes one language pair into consideration at a time. Minimally, to get a target translation of any source sentence one needs:

- A dictionary that will map each source word to an appropriate target word.

- Rules representing regular source sentence structure.

- Rules representing regular target sentence structure.

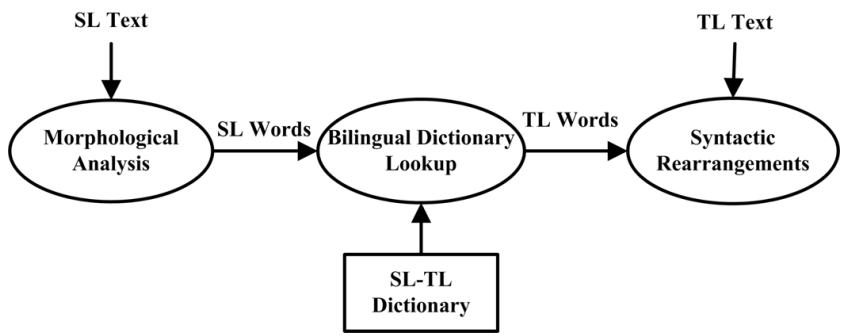

Fig. 2. Direct Machine Translation Approach

The most well-known Direct Machine Translation system for Indian languages is Anusaaraka [10], developed by IIT Kanpur which is now being continued at IIIT Hyderabad.

A direct translation system is appropriate for similar languages like Hindi and Punjabi [11], [12]. Vishal Goyal and Gurpreet Singh Lehel of Punjab University have developed a web based Hindi to Punjabi MT system with 95\% accuracy. Their system has additional modules for training the system for generating the lexicon using already existing corpus, input text font conversion into Unicode format to make the system free from specific font dependency.

Advantages of Direct MT

Advantages of using Direct MT are:

- Translation is usually comprehended by the reader with little effort.

- Translation is very effective for languages with similar grammar rules and structure.

- $\quad$ Systems are easy to implement.

Disadvantage of Direct MT

The Direct MT systems have following disadvantages.

- Direct MT involves only lexical analysis. It does not consider structure and relationships between words.

- Direct MT systems are developed for a specific language pair and cannot be adapted for different language pairs.

- $\quad$ Direct MT systems can be quite expensive, for multilingual scenarios [13].

- Some of the source text meaning can be lost in the translation. 
2) Interlingua Translation: Interlingual machine translation is another classical approach to machine translation. This is an alternative to less efficient direct translation approach and includes transfer approach. In this approach, the source language is transformed into an Interlingua, which is an intermediate abstract language-independent representation. Then target language is generated from this Interlingua. This approach is more efficient than direct translation as it is not merely a dictionary mapping of two languages. In this approach linguistic rules which are specific to the language pair transform the source language representation into an abstract target language representation and from this the target sentence is generated [14]. Figure 3 shows how different languages can be translated through this system.

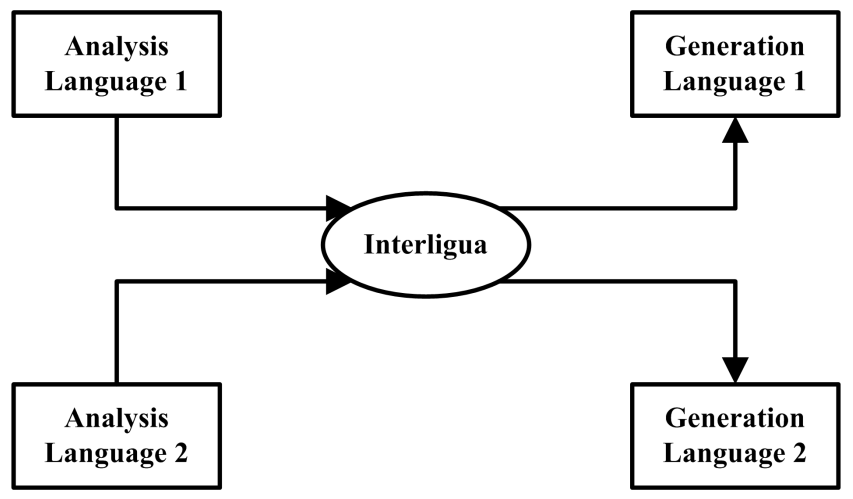

Fig. 3. Interlingua Translation [14]

\section{Advantages of Interlingua Machine Translation}

- It gives a meaning-based representation and can be used in applications like information retrieval.

- An Interlingua system has to resolve all the ambiguities so that translation to any language can take place from the Interlingua representation.

- $\quad$ The system is more practical when several languages are to be interpreted

Disadvantage of Interlingua Machine Translation

- Time efficiency of this system is lower than the Direct Machine Translation system.

- Major problem lies in defining a universal abstract (Interlingua) representation which preserves the meaning of a sentence.

3) Transfer Based Translation: In this translation system, a database of translation rules is used to translate text from source to target language. Whenever a sentence matches one of the rules, or examples, it is translated directly using a dictionary. The approach uses a dictionary to directly convert source into target whenever a sentence matches one of the transfer rules. Source language dictionary, Target language dictionary and a bilingual dictionary is used for this purpose. Figure 4 shows the approach of Transfer Based Translation.

Advantages of Transfer-Based MT Transfer-based approach has following advantages.

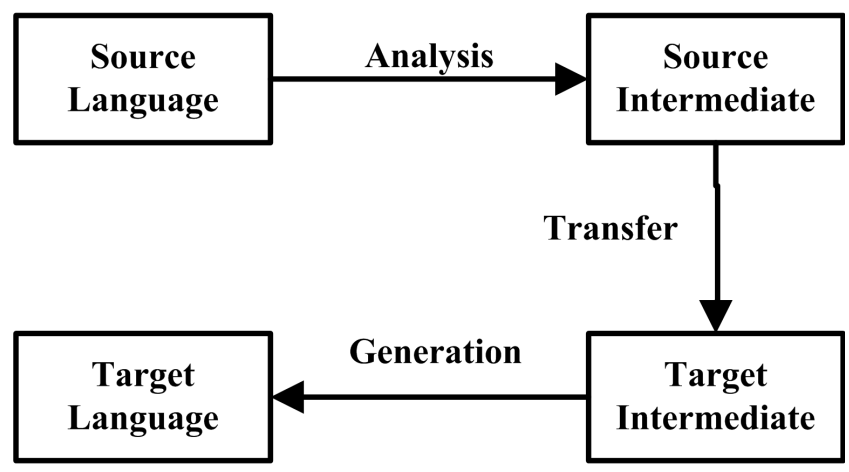

Fig. 4. Transfer Based Translation

- It has a modular structure.

- The system easily handles ambiguities that carry over from one language to another.

Disadvantage of Transfer-Based MT

Some of the source text meaning can be lost in the translation [13].

\section{B. Corpus Based Translation}

Corpus based Machine Translation is emerging as most used methodology for translation in today's world. It is based on statistical analysis of source and target language corpus. Huge dataset is required for initial statistical analysis. This can be approached by two different methods mentioned in sub sections below.

1) Statistical Machine Translation: Modern MT systems are deviating from classical approach of rule based translations. Translation process has been defined as a two stage process now. The Statistical Machine Translation (SMT) systems are based on the view that every sentence in a language has a possible translation in the target language. A sentence can be translated from one language to another in many possible ways. The system consists of three different models. In the first stage many output sentence candidates in the target language are generated. These candidates are stored in a lattice. This stage involves a bilingual translation lexicon, which gives possible translations for each word or phrase in the input sentence, with a probability assigned to every translation. The Language Model (LM) computes the probability of the target language $\mathbf{T}$ as probability $\mathrm{P}(\mathrm{T})$. In the second stage, a search component, called decoder, searches for the best output candidate according to a statistical scoring function. The Translation Model (TM), helps to compute the conditional probability of target sentences given the source sentence, $\mathrm{P}(\mathrm{T} I \mathrm{~S})$. Decoder maximizes the product of LM and TM probabilities as given in equation 1.The basic sketch of SMT system is shown in Figure 5.

$$
P(S, T)=\operatorname{argmax} P(T) * P(S \mid T)
$$

Though SMT systems are able to handle local re-orderings by themselves, in case of long-distance transformations they benefit considerably from external guidance.

Advantages of SMT: 


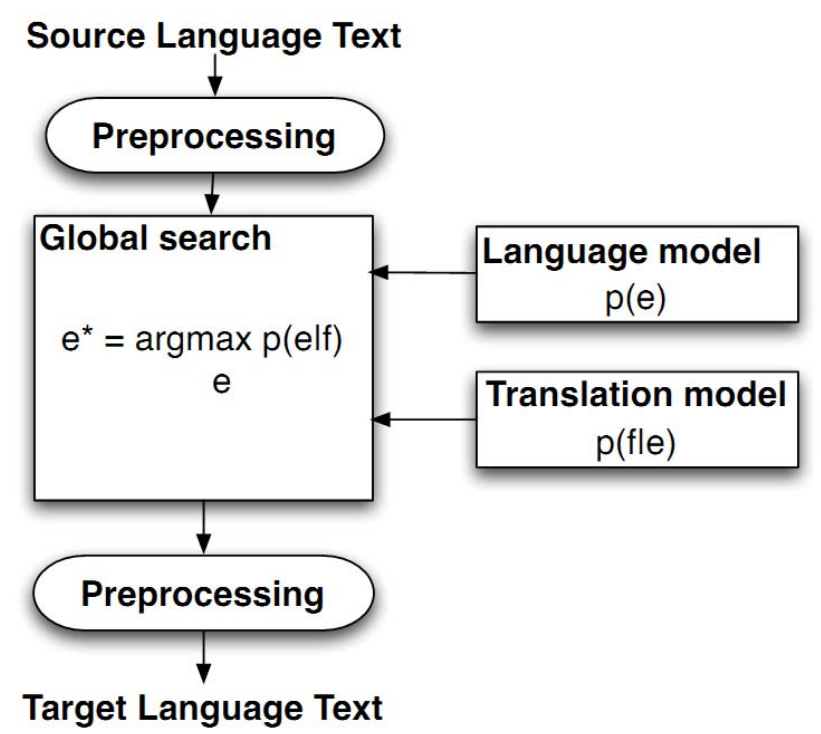

Fig. 5. Statistical Machine Translation

- There is a great deal of natural language in machinereadable format.

- Generally, SMT systems are not tailored to any specific pair of languages.

- Rule-based translation systems require the manual development of linguistic rules, which can be costly, and which often do not generalize to other languages.

Limitations of SMT:

- Corpus creation can be costly for users with limited resources.

- The results are unexpected. Superficial fluency can be deceiving.

- Statistical machine translation do not work well between languages that have significantly different word orders (e.g. Japanese and European languages).

- The benefits are overemphasized for European languages.

2) Example based Machine Translation: An Example based Machine translation system (EBMT) system maintains a corpus consisting of translation examples between source and target languages. An EBMT system has two modules: Retrieval module and an adaptation module. The retrieval module retrieves a similar sentence and its translation from the corpus for the given source sentence. The adaptation module then adapts the retrieved translation to get the final corrected translation. Consider the English to Hindi translation for the following sentence. Rohan eats a Mango. The retrieval module retrieves the following sentence and its translation from the corpus from a list of approximately matching sentences. It uses some similarity measures based on word similarity or syntactic and semantic similarity to identify this set of approximately matching sentences. From these the system selects the sentence with closest match with the input sentence.
If the system selects Seema eats a Mango and its translation Seema aam khaati hai as the closest one, it replaces Rohan with Seema and khaata with khaati and finally forms the translation. Seeta aam khaati hai.

Figure 6 shows a Translation Template of a phrase in two different languages.

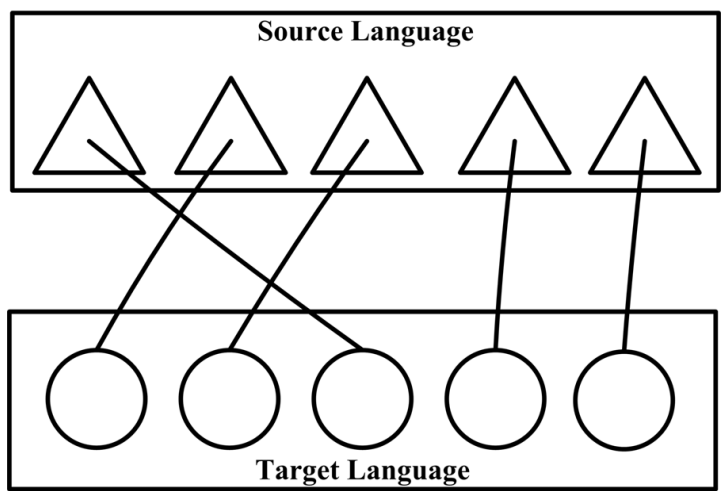

Fig. 6. Translation Template of a phrase in two different languages.

In this approach a simple adaptation is required to replace the word and suffix replacements. This method may not work in case of translation divergence where structurally similar sentences of the source language get translated into a different structure [15].

\section{Hybrid Machine Translation}

Recent advances in Machine Translation are based Hybrid machine translation. This method of machine translation utilizes multiple machine translation approaches within a single machine translation system thus making it a hybrid MTS. The objective behind development of these systems is to get best possible translation by exploiting the features of various existing approaches. There are various combinations under the category of hybrid machine translation approaches.

1) Statistical Rule Generation: In this hybrid approach we use statistical data to generate lexical and syntactic rules. The input is then processed with these rules as if it were a rulebased translator [27]. In this approach precious time is saved in by not creating a set of comprehensive, fine-grained linguistic rules by extracting those rules from the training corpus. But we still have some disadvantages as the accuracy of the translation will depend heavily on the similarity of the input text to the text of the training corpus. As a result, this technique has had the most success in domain-specific applications, and has the same difficulties with domain adaptation as many statistical machine translation systems [28]

2) Multi-Engine MT: The Multi-Engine MT (MEMT) architecture combines the outputs of multiple MT engines using a statistical language model of the target language. This approach to hybrid machine translation involves running multiple machine translation systems in parallel. The final output is generated by combining the output of all the sub-systems. It has been used successfully in a number of MT research systems, for both text and speech translation [29]. 
TABLE I. MAChinE TransLation SyStems IN INDIA

\begin{tabular}{|c|c|c|c|c|c|}
\hline System & Approach used & Target Language & Year & Place & Features \\
\hline Anusaaraka [10] & Direct & $\begin{array}{l}\text { Bengali, Kannada, } \\
\text { Marathi, Punjabi, } \\
\text { Telegu to Hindi }\end{array}$ & 1995 & $\begin{array}{l}\text { Started in IIT Kan- } \\
\text { pur, continuing in } \\
\text { IIIT, Hyderabad }\end{array}$ & $\begin{array}{l}\text { Uses Paninian grammar and matches local words } \\
\text { between source and target language. }\end{array}$ \\
\hline $\begin{array}{l}\text { Hindi to Punjabi MTS } \\
\text { [16] }\end{array}$ & Direct & Hindi to Punjabi & 2007 & $\begin{array}{l}\text { Punjab University, } \\
\text { Patiala }\end{array}$ & $\begin{array}{l}\text { Morphological analysis, word sense disambigua- } \\
\text { tion, post processing and transliteration }\end{array}$ \\
\hline Mantra [17] & Transfer Based & $\begin{array}{l}\text { English to Hindi, } \\
\text { Gujrati, Telegu. } \\
\text { Hindi to English, } \\
\text { Bengali, Marathi }\end{array}$ & 1995 & CDAC,Pune & Uses Tree Adjoining Grammar Formalism. \\
\hline Matra [18] & Transfer Based & English to Hindi & 2004 & CDAC,Pune & $\begin{array}{l}\text { Human assisted translation project uses rule bases } \\
\text { and heuristics. }\end{array}$ \\
\hline Shakti [19] & Transfer Based & $\begin{array}{l}\text { English to Hindi, } \\
\text { Marathi, Telegu }\end{array}$ & 2004 & $\begin{array}{l}\text { IISc Bangalore and } \\
\text { IIIT, Hyderabad }\end{array}$ & $\begin{array}{l}\text { Works by combining rule based and statistical } \\
\text { approach. }\end{array}$ \\
\hline Anubaad [20] & Transfer Based & English to Bengali & 2005 & CDAC, Kolkata & $\begin{array}{l}\text { Hybrid system which uses n-gram approach for } \\
\text { POS tagging. Works at sentence level }\end{array}$ \\
\hline Sampark [21] & Transfer Based & $\begin{array}{l}\text { Punjabi-Hindi, } \\
\text { Telegu to Tamil, } \\
\text { Hindi-Urdu, Hindi } \\
\text { to Telegu }\end{array}$ & 2005 & IIIT Hyderabad & $\begin{array}{l}\text { Uses Computational Paninian Grammar (CPG) } \\
\text { approach for analyzing language and combines it } \\
\text { with machine learning }\end{array}$ \\
\hline Anglabharti [22] & Interlingual & $\begin{array}{l}\text { English to Hindi, } \\
\text { Tamil }\end{array}$ & 1991 & IIT,Kanpur & $\begin{array}{l}\text { Uses intermediate structure Pseudo Lingua for } \\
\text { Indian Languages. }\end{array}$ \\
\hline AnglaHindi [23] & Interlingual & English to Hindi & 1991 & IIT,Kanpur & $\begin{array}{l}\text { Uses rule-bases, example-base and statistics to } \\
\text { obtain translation for frequently encountered noun } \\
\text { and verb phrasal. }\end{array}$ \\
\hline $\begin{array}{l}\text { UNL English Hindi MTS } \\
\text { [24] }\end{array}$ & Interlingual & English to Hindi & 2001 & IIT, Mumbai & Uses Universal Natural Language as interlingua \\
\hline English Hindi MTS & $\begin{array}{l}\text { Statistical Machine Transla- } \\
\text { tion }\end{array}$ & English to Hindi & 2010 & IIIT, Hyderabad & $\begin{array}{l}\text { Combines Rule Based Machine Translation and } \\
\text { phrase based SMT }\end{array}$ \\
\hline English Malyalam MTS & $\begin{array}{l}\text { Statistical Machine Transla- } \\
\text { tion }\end{array}$ & $\begin{array}{l}\text { English } \\
\text { Malyalam }\end{array}$ & 2010 & $\begin{array}{l}\text { Cochin University } \\
\text {,Cochin }\end{array}$ & $\begin{array}{l}\text { Uses SMT by using monolingual Malayalam cor- } \\
\text { pus and a bilingual English/Malayalam corpus in } \\
\text { the training phase }\end{array}$ \\
\hline Hindi-English MTS & $\begin{array}{l}\text { Statistical Machine Transla- } \\
\text { tion }\end{array}$ & English to Hindi & 2010 & $\begin{array}{l}\text { State University of } \\
\text { New York. }\end{array}$ & $\begin{array}{l}\text { Combines Rule Based Machine Translation and } \\
\text { phrase based SMT }\end{array}$ \\
\hline Kriya [6] & $\begin{array}{l}\text { Statistical Machine Transla- } \\
\text { tion }\end{array}$ & Hindi-English & 2012 & $\begin{array}{l}\text { Simon Fraser Uni- } \\
\text { versity }\end{array}$ & It is hierarchical phrase-based (hiero) SMT system \\
\hline Vaasaanubaada [25] & $\begin{array}{l}\text { Example Based Machine } \\
\text { Translation }\end{array}$ & Bengali Assamese & 2002 & $\begin{array}{l}\text { Pondichery Univer- } \\
\text { sity }\end{array}$ & $\begin{array}{l}\text { Preprocessing and post processing task, longer } \\
\text { sentences fragmented at punctuation, backtracking } \\
\text { for unmatched results. }\end{array}$ \\
\hline Anubharti [26] & $\begin{array}{l}\text { Example Based Machine } \\
\text { Translation }\end{array}$ & Hindi-English & 2004 & IIT,Kanpur & $\begin{array}{l}\text { Hybrid Example based system which combines } \\
\text { pattern based and example based approach. }\end{array}$ \\
\hline Shiva & $\begin{array}{l}\text { Example Based Machine } \\
\text { Translation }\end{array}$ & Hindi-English & 2004 & $\begin{array}{l}\text { IISc Bangalore,IIIT } \\
\text { Hyderabad }\end{array}$ & $\begin{array}{l}\text { Uses linguistic rules and statistical approach to } \\
\text { infer linguistic information. }\end{array}$ \\
\hline
\end{tabular}

\section{MaChine Translation Systems In India}

Machine Translation is an active topic of research in India from 1991 onwards. The first work started at IIT Kanpur and now it has spread to many Universities. There have been various Government sponsored projects to develop these systems for Indian languages. Different research groups have worked on different types of approaches discussed above for their system implementations. Based on different approaches, various MTS are listed in Table 1.

\section{CONCLUSION}

In this paper we have reviewed various Machine Translation approaches and existing Machine Translation systems in India. Various MT groups have used different formalisms best suited to their applications. Of them transfer based systems are more flexible and it can be extended to language pairs in a multilingual environment. Most of the MT systems are for Hindi and there are only very few systems for south Indian languages. More research has to be done in these areas to overcome the language barrier faced by India. The MT systems so far developed have many shortcomings in terms of rule set, dictionary, translation methodology and it is apparent from the survey that further work is needed in MT as a whole to produce intelligible translations.

\section{REFERENCES}

[1] D. D. Lewis and K. S. Jones, "Natural language processing for information retrieval," Communications of the ACM, vol. 39, no. 1, pp. 92-101, 1996.

[2] D. D. Rao, "Machine translation," Resonance, vol. 3, no. 7, pp. 61-70, 1998.

[3] K. Keniston and D. Kumar, IT experience in India: Bridging the digital divide. Sage, 2004.

[4] B. Murthy and W. Deshpande, "Language technology in india: past, present and future, 1998," 2011.

[5] V. Goyal and G. S. Lehal, "Advances in machine translation systems." Language In India, vol. 9, no. 11, 2009.

[6] B. Sankaran, M. Razmara, and A. Sarkar, "Kriya-an end-to-end hierarchical phrase-based mt system," The Prague Bulletin of Mathematical Linguistics, vol. 97, pp. 83-98, 2012.

[7] S. Raman and N. Alwar, "An ai-based approach to machine translation in indian languages," Communications of the ACM, vol. 33, no. 5, pp. 521-527, 1990.

[8] K. Papineni, S. Roukos, T. Ward, and W.-J. Zhu, "Bleu: a method for automatic evaluation of machine translation," in Proceedings of the 40th annual meeting on association for computational linguistics. Association for Computational Linguistics, 2002, pp. 311-318.

[9] N. Mandelblit, "Machine translation: A cognitive linguistics approach," in Proceedings of the Fifth International Conference on Theoretical and Methodological Issues in Machine Translation, Kyoto, Japan, 1993.

[10] A. Bharati, V. Chaitanya, A. P. Kulkarni, and R. Sangal, "Anusaaraka: Machine translation in stages," VIVEK-BOMBAY-, vol. 10, pp. 22-25, 1997.

[11] G. S. Josan and G. S. Lehal, "A punjabi to hindi machine transla- 
tion system," in 22nd International Conference on on Computational Linguistics: Demonstration Papers. Association for Computational Linguistics, 2008, pp. 157-160.

[12] V. Goyal and G. S. Lehal, "Evaluation of hindi to punjabi machine translation system," arXiv preprint arXiv:0910.1868, 2009.

[13] S. K. Dwivedi and P. P. Sukhadeve, "Machine translation system in indian perspectives," Journal of computer science, vol. 6, no. 10, p 1111, 2010.

[14] S. Dave, J. Parikh, and P. Bhattacharyya, "Interlingua-based englishhindi machine translation and language divergence," Machine Transla tion, vol. 16, no. 4, pp. 251-304, 2001.

[15] D. Gupta and N. Chatterjee, "Identification of divergence for english to hindi ebmt," in Proceeding of MT Summit-IX, 2003, pp. 141-148.

[16] V. Goyal and G. S. Lehal, "Web based hindi to punjabi machine translation system," Journal of Emerging Technologies in Web Intelligence. vol. 2, no. 2, pp. 148-151, 2010.

[17] H. Darbari, "Computer-assisted translation system-an indian perspective," Machine Translation Summit VII, 13th-17th September, pp. 80-85, 1999.

[18] R. Ananthakrishnan, M. Kavitha, J. H. Jayprasad, R. S. Chandra Shekhar, and S. M. Sawani Bade, "Matra: A practical approach to fully-automatic indicative english-hindi machine translation," in Symposium on Modeling and Shallow Parsing of Indian Languages (MSPIL'06), 2006.

[19] R. Sangal, "Architecture of shakti machine translation system," IIIT Hyderabad, 2004.

[20] S. Bandyopadhyay, "Anubaad-the translator from english to indian languages," in Proceedings of the 7th State Science and Technology Congress,(SSTCâĂŹO0), Calcutta, India, 2000, pp. 1-9.

[21] R. Ahmad, P. Kumar, B. Rambabu, P. Sajja, M. K. Sinha, and R. Sangal,
"Enhancing throughput of a machine translation system using mapreduce framework: An engineering approach," ICON, 2011.

[22] R. Sinha, K. Sivaraman, A. Agrawal, R. Jain, R. Srivastava, and A. Jain, "Anglabharti: a multilingual machine aided translation project on translation from english to indian languages," in Systems, Man and Cybernetics, 1995. Intelligent Systems for the 21st Century., IEEE International Conference on, vol. 2. IEEE, 1995, pp. 1609-1614.

[23] R. Sinha and A. Jain, "Anglahindi: an english to hindi machine-aided translation system," MT Summit IX, New Orleans, USA, pp. 494-497, 2003.

[24] C. Boitet, P. Bhattacharyya, E. Blanc, S. Meena, S. Boudhh, G. Fafiotte, A. Falaise, and V. Vacchani, "Building hindi-french-english-unl resources for survitra-cifli, a linguistic survival system under construction," in Seventh international symposium on natural language processing, 2007, p. 7.

[25] K. Vijayanand, S. I. Choudhury, and P. Ratna, "Vaasaanubaada: automatic machine translation of bilingual bengali-assamese news texts," in Language Engineering Conference, 2002. Proceedings. IEEE, 2002, pp. 183-188.

[26] R. Jain, R. Sinha, and A. Jain, "Anubharti-using hybrid example-based approach for machine translation," STRANS-2001, IIT Kanpur, pp. 2032, 2001.

[27] J. Hutchins, "Machine translation: A concise history," Computer aided translation: Theory and practice, 2007.

[28] J.-S. Chang and K.-Y. Su, "Corpus-based statistics-oriented (cbso) machine translation researches in taiwan," AMTA (1997), pp. 165-173, 1997

[29] C. Hogan and R. E. Frederking, "An evaluation of the multi-engine $\mathrm{mt}$ architecture," in Machine Translation and the Information Soup. Springer, 1998, pp. 113-123. 